\title{
Research on Information Fusion Frame and Its Military Applications
}

\author{
Zhang Minghu ${ }^{1, a}$, Yang Hongyu ${ }^{1, b}$, Bai Xuelian ${ }^{1, a}$ and Chen Hongmin ${ }^{2, b}$ \\ ${ }^{1}$ Dept. of Underwater Weaponry and Chemical Defense, Dalian Naval Academy, Dalian, China \\ ${ }^{2}$ The Detachment of Warship Training, Dalian Naval Academy, Dalian, China \\ azmhly20070618@163.com, bmhly20040318@ sina.com
}

Keywords: Information fusion, system frame, model construction, military application.

\begin{abstract}
For the sake of implementing effectively detection and rapid exact identification for the chemical agents in sea-battlefield, the automatic detection technique using the neural network(NN) information fusion for the chemical agents, is studied. Firstly, the information fusion treatment model and system frame are analyzed; Secondly, the modeling means and chemical agents feature extraction are studied, connecting the wavelet analysis with the NN organically, and based on the wavelet transfer and NN, the system of the speedy features extraction and identification for chemical agents, the NN distinguishing chemical agent system, is founded; Thirdly, the hardware realization and software component are introduced in brief; Lastly, the method is workable, and of the high identification accuracy, remarkable generalization capability, good stability, fast speed, and high reliability. And the method has the general military application value.
\end{abstract}

\section{Introduction}

The conception of information fusion began in 1970s' initial stages, rooted in the requirement of the $\mathrm{C}^{3} \mathrm{I}$ (Command, Control, Communication and Intelligence) system in military domain, then intituled the multi-sources correlation or multi-sensors mix data fusion, and the technologies were established in 1980s. The JDL(Joint Directors of Laboratories) of the DOD(department of defense) of the US defined information fusion as such process from military application: those data and information from multi-sensors and multi-info-sources add association, correlation and combination, to obtain the accurate position estimation, identity estimation, and the proper integrity estimation for the battlefield instance and menace and its importance degree. Waltz and Llinas put up supplement and modification the above definition: the information fusion is a sort of multilayer and/or multiside treatment process, the process is detection, association, correlation, estimation and combination to obtain the accurate state estimation, identity recognition, the whole situation estimation and menace estimation.

\section{Treatment model}

The information fusion treatment model is for a set of the treatment processes' description, which is the constitution of the system function units, not refered the physics frame and software realization of each of the units, and the treatment processes also allow feedback, viz regulate the fusion frames based on the decision-making. Fig. 1 is the information fusion treatment model that was put forward by the data fusion work group in the US, which it plays important influence to understand the basic conception of the information fusion.

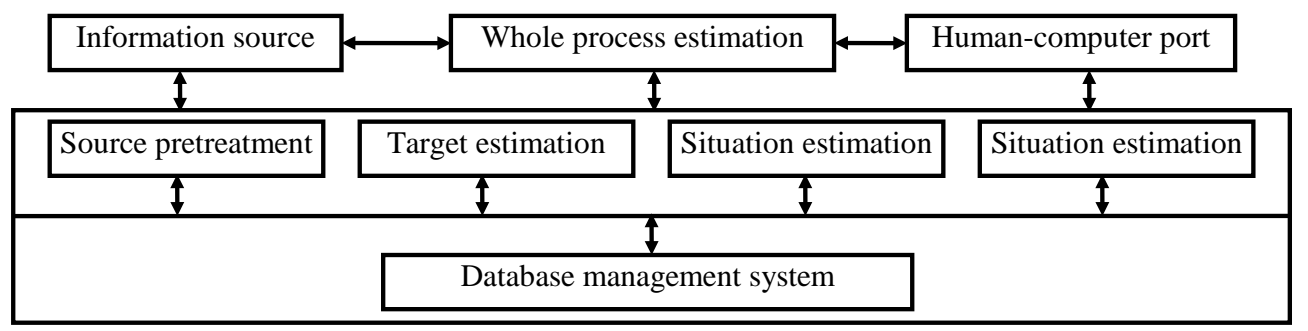

Fig.1 Information fusion treatment model 


\section{The information fusion system frame}

The research of the system frame of the information fusion includes two parts: hiberarchy and architecture. The hiberarchy mostly based on the information to analyze the fusion system; and the architecture mostly based on the hardware.

Hiberarchy. The information fusion system may be compartmentalized according to administrative levels, but there are more opinions. At present, the universal acceptant opinion is three layers fusion structure, namely data layer, feature layer and decision-making layer.

The hiberarchy of the information fusion is compartmentalized according to the information abstract degree. In the practice engineering application of the multi-sensors information fusion (MSIF) system, the hiberarchy model, which was established and adopted, should synthetically consider the sensors performance, system calculation ability, communications bandwidth, expectation nicety rate, and existence fund power. Moreover, the establishment of the hiberarchy based on the information can better confirm the architecture based on the system hardware.

Architecture. The system hardware architecture are approximately divided to three species: concentration style, distribution style and admixture style.

The architecture adopted is entirely the various practice requirement. When the architecture is designed, it's confirmed by the established system hiberarchy, at the same time, the many supportion technologies must also be considered, such as data traffic, database management, human-computer port, and sensor management.

\section{Military application model construction}

Neural network (NN) is a new technology for pattern recognition, which can distinguish nonlinear complex objects using self-adapting mode. It has advantages of high legitimacy and strong anti-interference capacity. At presents, there are many applied studies on the BP NN and RBF NN in the chemical agents and environment monitoring, the BP is the most comprehensive application in the aspect of spectrum discrimination and is the most successful technology, and appears many improved $\mathrm{BP}$ algorithm. And the RBF NN has become the research hotspot at present, which has higher discrimination capability and faster learning speed. Kohonen network and adaptive resonance NN are belonging to self-organizing and self-adapting NN, which can cultivate independent learning ability, and be applied in the adaptability training of the equipment to new surroundings. NN have high recognition capability and mature theory, and the algorithm is complex, only combined with the method of the efficient feature extraction or selection, can we get ahead in the practical application.

Modeling means. Takes example for the naval ships chemical detection (NSCD), for the sake of implementing effectively detection and rapid exact identification for the chemical agents in sea-battlefield, the automatic detection technique -NN information fusion technology- for the chemical agents, is studied. The model of the NN information fusion system is built. At the same time, connecting the wavelet analysis with the NN organically, and based on the wavelet transfer and NN, the system of the speedy features extraction and identification for chemical agents, the neural network distinguishing chemical agent (NNDCA) system, is founded. In order to establish the NSCD system of high speed decision-making, advanced principle, higher precision and sensitivity, automation, and intelligence, and heightening the NSCD technology.

Selecting the sample data of the chemical agents to carry on the feature extraction, and this is the premise of the selecting recognition parameter and the structuring distinguishing NN. Carries on the wavelet energy spectrum analysis for the chemical agents sample parameter, to obtain the corresponding wavelet energy spectrum characteristic vector of the parameter residual error data, which are available in structuring the study sample of the recognition network.

Founded on certain quantity training samples collection (usually is the symptom, chemical agent of data set), to carry on the training for the NN to obtain the expectation recognition network, then to comply with the current recognition input, the chemical agent is distinguished for the system. Before study and recognition, it usually does justice to the primary distinguishing data and the training 
sample data. The goal is to provide the appropriate recognition input for the recognition network and the training sample, and the usable feature vector for the NN distinguishing. When the chemical agent enters the chemical sensors, the complex film in the sensors adsorb the chemical agent molecule. According to the differences of the chemical agent compound, it may cause the resonance frequencies change through the mini-sensors. The mini-sensors have the different frequency feature response signal for the different chemical agent. This kind of response is recorded and transmitted to the NN, which has trained and joins the chemical sensors, to examine the hairlike distinction in these signals. The chemical agent feature is recorded in the database with comparison, distinction and confirmation the chemical agent type.

The NNDCA for the NSCD system based on the MSIF is to extract or select the feature of the chemical agent in sea battlefield, according as the feature of the every chemical agent which is measured by the multi-sensors in the system, and make use of the NN' ability of the non-linear mapping and pattern distinguishing, eventually distinguish the chemical agent to be measured by synthesis and analysis. Before using the $\mathrm{NN}$ to distinguish the chemical agents, the $\mathrm{NN}$ is trained to anticipant recognition network by some quantitative training samples sets.

The NNDCA was built up by the numerical value calculating, NN's learning and training. The input network's node-number is the dimension of the input characteristic vectors; the output's is amount of the chemical agents; the middle-tier's should be selected by the request of the training sample-set size and training error. The precondition of the distinguishing preferences and constructing recognition $\mathrm{NN}$ is the characteristic extracting for these samples data, the eigenvector of the normalized wavelet energy distribution can be obtained by the analyzing wavelet energy distribution for the data of the chemical agent sample parameters, and which may be regarded as the calculation basis of the parameter recognition confidence-degree or samples comprehensive discrete degree. The corresponding wavelet energy distribution eigenvector of the parameter residual data can be obtained by treating the chemical agent sample residual data, which can construct the learning sample of the recognition NN. If the training effect is imperfect, it need readjust the parameters of the $\mathrm{NN}$, repartition the training samples, and retrain the NN, till attain to the satisfying effect.

Chemical agents feature extraction. In the process of distinguishing the chemical agents, we can get the practical data of many parameters by chemical sensors. These data contain rich information of the chemical agents. Feature extraction or selection is the powerful tool to reduce the mode dimension. Reducing dimension of the primitive features can help minimize the wrong recognition rate of the classified device. Via wavelet package analysis technology, we can record the signals energy data of various frequencies, thus we get the frequency energy feature, which distinguishes one from another. Choosing feature parameter is based on choosing the sample data's features. With parameter features' comprehensive discrete degree or recognition credit degree as a criterion, we can get a group of better feature parameters to recognize. We have known that the different chemical agents obtain the different frequency energy feature vectors. They reflect the different chemical agents, and which provides the practical feature vectors for the recognition of chemical agents based on the NN. According to the feature vector to work out the sort decision-making, i.e., to confirm the chemical agent that it is which kind of chemical agents.

Hardware realization. In order to realize the NNDCA, to heighten the stability and reliability of the NSCD system, and to adapt to the requirement of miniaturization, the calculating method of the NNDCA should be transplanted to the chip of the hardware, and get the practical application. For the interface-control part of the NNDCA and NSCD system, the embedded designing thought may be used, the various single-chip or embedded computers can be adopted as the running platform. For the system of the biggish data quantity, it can adopt the DSP device to realize the beginning pretreatment function, and the embedded computer to realize the data traffic and interface management of the subassemblies in the NNDCA classifiers and NSCD system. In order to make the system run speedily and obtain the finer real-time ability, the NN classifiers can also adopt the realization project of the digital circuit based on FPGA. 
Software component. The software exploitation of the NNDCA is the quite complex software engineering. The software is mostly composed of the many modules, such as the chemical agent recognition process evaluation module, NNDCA module, data processing module, data management module, maintenance module, and database. The module of the NNDCA and the database are the cores of the wholly formed software.

\section{Conclusions}

Using the information fusion and wavelet signal means to distinguish the chemical agent, which the conclusions are as follows:

(1) The multi-dimensional information of the chemical agents can be obtained by using the measurement system based on the multi-sensor fusion; the data from the multi-sensor can be fused by the neutral networks, the results show that the method can remarkably fall the effects of the factors, interference, concentration, and condition, etc, for the measurement results, and heighten the accuracy and credibility of the measurement results.

(2) The anticipant recognition NN can be gained by training, testing, and verifying, on the basis of the relatively processing for the learning sample data and its feature extraction or selection, it is completely feasible that the analyses for the chemical agents with the NNDCA system based on the MSIF technology.

\section{Acknowledgements}

This work was financially supported in part by the national defense Basic Research Foundation of China (435B956), the National Defense Pre-Research Found of China(41101050403), and the $3^{\text {rd }}$ Academic Pre-Research Funds of Dalian Naval Academy of the 2110 Project (DLJY-XY2015004).

\section{References}

[1] G. Zhang. Chemical sensors, No. 1(2011), p. 54.

[2] Q. Pang, X. Yu, Y. Cheng, et al. Acta Automatica Sinica, No.4 (2003), p. 599.

[3] M. Zhang, G. Pang, H. Liu, et al. Journal of Guangxi Normal University: Natural Science Edition, No.1 (2008), p. 220.

[4] G. Wang, Zh. Zhang. Electrical Measurement \& Instrumentation, No. 2(2006), p. 1.

[5] H. Zhou. Journal of Chifeng University (Natural Science Edition), No. 5(2011), p. 96.

[6] F. Wang, Sh. Huang, Zh. Han. Aeronautical Computing Technique, No. 1(2009), p. 102.

[7] Y. Ao, B. Wang. Machine Tool \& Hydraulics, No. 13(2014), p. 168.

[8] J. Liu, P. Wang, L. Yang. Journal of Beijing University of Technology, No. 6(2014), p. 843.

[9] X. Tian, Zh. Ma, Sh. Tan. Journal of Sichuan Ordnance, No. 5(2015), p. 90.

[10] X. Jian, H. Jia, L. Shi. Chinese Journal of Construction Machinery, No. 2(2009), p. 227.

[11]H. Ge, L. Tian. Application Research of Computers, No. 1(2009), p. 19.

[12] M. Liu, L. Peng. Microelectronics \& Computers, No. 1(2009), p. 166. 